\title{
Supporting Information: Are RAFT and ATRP Universally Interchangeable Polymerization Methods in Network Formation?
}

Julia Cuthbert ${ }^{\sharp a}$, Shiwanka V. Wanasinghe ${ }^{\sharp b}$, Krzysztof Matyjaszewsk ${ }^{*}{ }^{* a}$, Dominik

Konkolewicz,,$b$

a Department of Chemistry, Carnegie Mellon University, 4400 Fifth Ave, Pittsburgh, PA, 15213 USA

${ }^{\mathrm{b}}$ Department of Chemistry and Biochemistry, Miami University, 651 E High St Oxford, OH,

$$
\begin{aligned}
& \text { 45056, USA } \\
& \ddagger \text { Co-first authors }
\end{aligned}
$$

*Corresponding authors: KM: matyjaszewski@cmu.edu, DK: d.konkolewicz@miamiOH.edu

Scheme S1. The synthesis of DSDA

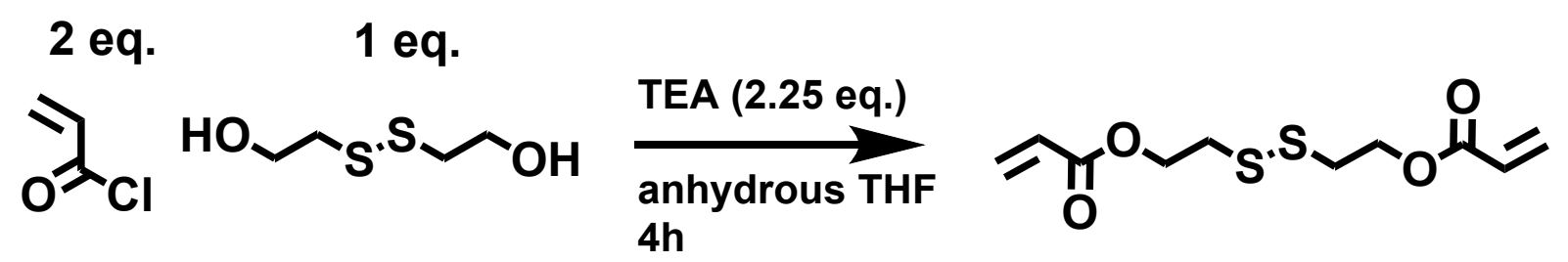

Synthesis of the Disulfide Diacrylate (DSDA) or 2,2'-dithiodiethanol diacrylate 
Bis(2-hydroxyethyl) disulfide (BHEDS, $1.1 \mathrm{~g}, 7.14 \mathrm{mmol}, 1.0$ equiv) and triethylamine (7.1 mL, $57.14 \mathrm{mmol}, 8.0$ equiv) were dissolved in dry THF $(22 \mathrm{~mL})$ in a $250 \mathrm{~mL}$ round bottom flask containing a magnetic stir bar. This flask was immersed in an ice bath for $15 \mathrm{~min}$, and then acryloyl chloride (2.6 g, $28.57 \mathrm{mmol}, 4$ equiv) was added dropwise while stirring. The resulting solution was stirred at $20{ }^{\circ} \mathrm{C}$ for $24 \mathrm{~h}$. THF was removed by evaporation under reduced pressure and the crude product was dissolved in $22 \mathrm{~mL}$ of chloroform. This solution was washed three times with an aqueous $0.1 \mathrm{M} \mathrm{K}_{2} \mathrm{CO}_{3}$ solution followed by five washings with deionized water, three washings with $\mathrm{HCL}(0.1 \mathrm{M})$ and two washings with $\mathrm{NaCl}$ solution. The purified organic solution was dried using anhydrous $\mathrm{MgSO}_{4}$, and the chloroform was removed under reduced pressure. The final disulfide-based diacrylate product was obtained as a light-yellow oil ( $1.1 \mathrm{~g}, \approx 60 \%$ yield). Chemical shift values for the compound - 6.40-6.50 and 5.80-5.90 ppm (4H, doublet, $\left.\mathrm{CH}_{2}-\mathrm{CHCOO}\right), 6.05-$ $6.20 \mathrm{ppm}\left(2 \mathrm{H}\right.$, multiple, $\left.\mathrm{CH}_{2} \mathrm{CHCOO}\right), 4.35-4.45 \mathrm{ppm}\left(4 \mathrm{H}\right.$, triplet, $\mathrm{COOCH}_{2} \mathrm{CH}_{2}$ ), and 2.90-3.00 ppm (4H, triplet, $\mathrm{COOCH}_{2} \mathrm{CH}_{2} \mathrm{~S}$ ) (Figure S1). A note of caution: BHEDS is highly toxic and poisonous. Please use care when handling and consult the SDS information.

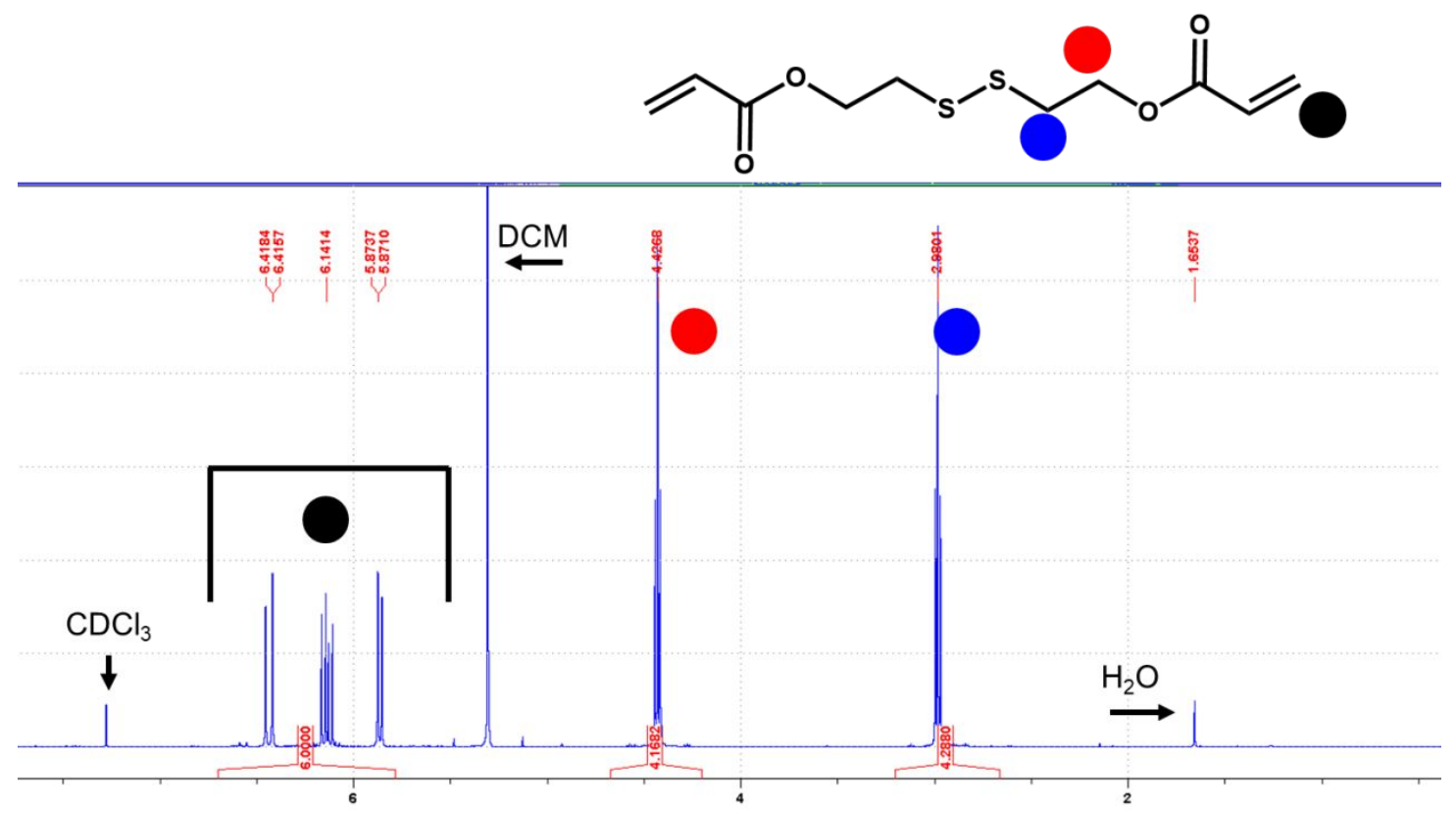

Figure S1. The ${ }^{1} \mathrm{H}$ NMR spectrum of DSDA in $\mathrm{CDCl}_{3}$. Peaks and integrations are shown. 


\section{Experimental Details for the Synthesis of the ATRP Linear Polymer Models}

Scheme S2. The linear polymer models prepared by ATRP
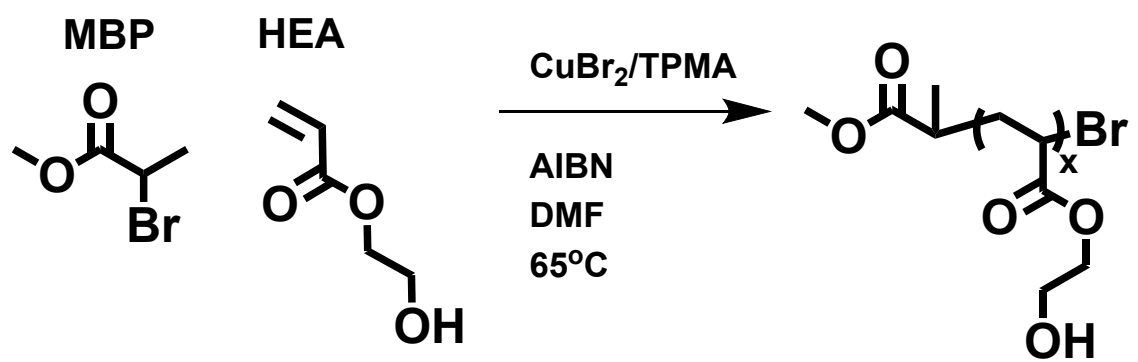

General Synthesis Procedure for the ATRP Linear Polymer Models

To a Schlenk flask, HEA, $\mathrm{CuBr}_{2}$, TPMA, AIBN, and DMF were added. While stirring, the solution was sparged with nitrogen gas for 30 min. Under positive nitrogen pressure, MBP in DMF was added to the pre-gel solution. The Schlenk flask was sealed and placed in an oil bath $65^{\circ} \mathrm{C}$ for 24 $\mathrm{h}$ to perform ICAR ATRP. The reaction was stopped by exposure to oxygen and cooling. The conversion of monomer was determined by ${ }^{1} \mathrm{H}$ NMR, which was used to calculate the theoretical molecular weight $\left(\mathrm{M}_{\mathrm{n} \text {,theo }}\right)$. The apparent molecular weight $\left(\mathrm{M}_{\mathrm{n}, \mathrm{app}}\right)$ was determined by GPC (DMF as the solvent). For the formulation details for each linear polymer model (target DP 100, 200, and 500) see Table S1.

Table S1. The materials used for the ATRP linear polymer models for the three target DPs (100, 200, and 500) and the concentrations of each material (molarity [M]). ${ }^{1}$

\begin{tabular}{|c|c|c|c|c|c|}
\hline Target $\mathrm{DP}^{2}$ & [HEA] & [MBP] & {$\left[\mathrm{Cu}(\mathrm{II}) \mathrm{Br}_{2}\right]$} & [TPMA] & [AIBN] \\
\hline $\mathbf{A} 100$ & 4.35 & $4.4 \times 10^{-2}$ & $8.7 \times 10^{-4}$ & $3.5 \times 10^{-3}$ & $8.7 \times 10^{-3}$ \\
\hline $\mathbf{A} 200$ & 4.35 & $2.2 \times 10^{-2}$ & $4.4 \times 10^{-4}$ & $1.7 \times 10^{-3}$ & $4.4 \times 10^{-3}$ \\
\hline
\end{tabular}




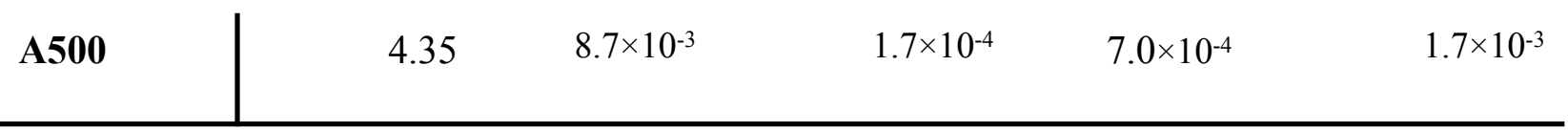

1) Solvent = DMF; $V_{S}=V_{M}$. 2) The target degree of polymerization with "A" to designate ATRP as the method used. Therefore, the molar equivalents of monomer to chain end (MBP) are as follows: $100 / 1 ; 200 / 1$; and 500/1.

\section{Experimental Details for the Synthesis of the RAFT Linear Polymer Models}

Scheme S3. The linear polymer models prepared by RAFT polymerization.

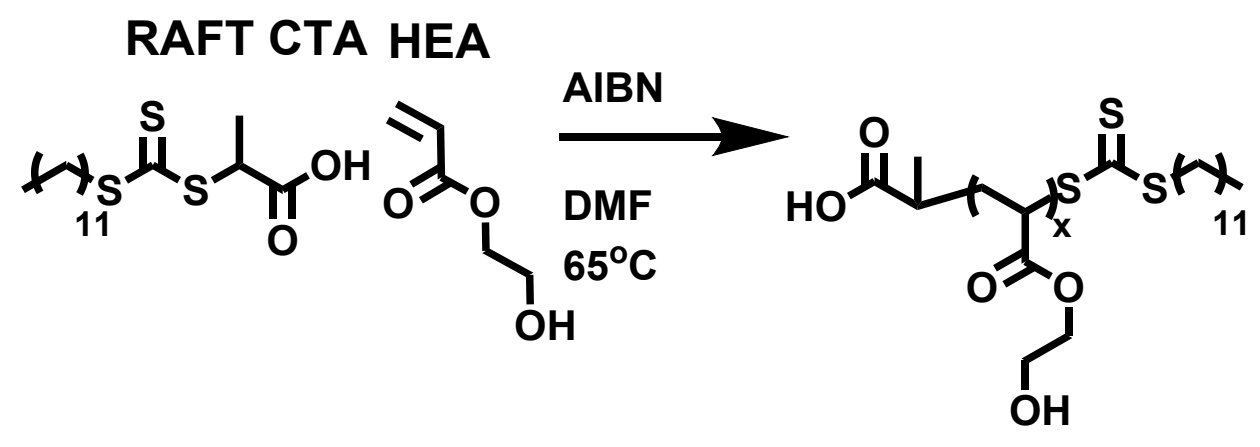

General Procedure for the Synthesis of RAFT Linear Polymer Models

To a Schlenk flask, HEA, PADTC, AIBN, and DMF were added. While stirring, the solution was sparged with nitrogen gas for $30 \mathrm{~min}$. The Schlenk flask was sealed and placed in an oil bath $70^{\circ} \mathrm{C}$ for $24 \mathrm{~h}$ to perform RAFT polymerization. The reaction was stopped by exposure to oxygen and cooling. The conversion of monomer was determined by ${ }^{1} \mathrm{H}$ NMR, which was used to calculate $\mathrm{M}_{\mathrm{n} \text {,theo }}$ and the $\mathrm{M}_{\mathrm{n} \text {,app }}$ was determined by GPC (DMF as the solvent). For the formulation details for each linear polymer model (target DP 100, 200, and 500) see Table S2.

Table S2. The materials used for the ATRP linear polymer models for the three target DPs (100, 200 , and 500) and the concentrations of each material (molarity $[\mathrm{M}])^{1}$ 


\begin{tabular}{l|ccc}
\hline R100 & 4.35 & $4.4 \times 10^{-2}$ & $8.7 \times 10^{-3}$ \\
R200 & 4.35 & $2.2 \times 10^{-2}$ & $4.4 \times 10^{-3}$ \\
$\mathbf{R 5 0 0}$ & 4.35 & $8.7 \times 10^{-3}$ & $1.7 \times 10^{-3}$ \\
\hline
\end{tabular}

1) Solvent = DMF; $V_{S}=V_{M}$. 2) The target degree of polymerization with "R" to designate RAFT polymerization as the method used. Therefore, the molar equivalents of monomer to chain end (PADTC) are as follows: 100/1; 200/1; and 500/1.

Table S3. The RDRP linear polymer models were compared by the conversion of vinyl bonds and the SEC characterization.

\begin{tabular}{|c|c|c|c|c|}
\hline Target DP & $\begin{array}{l}\text { Conv. Vinyl } \\
\text { Bonds (\%) }\end{array}$ & $M_{n, \text { theo }} 4$ & $M_{\text {n,app }} 5$ & $\mathrm{D}^{6}$ \\
\hline A100 & $>95$ & $1.17 \times 10^{4}$ & $1.36 \times 10^{4}$ & 1.28 \\
\hline R100 & $>95$ & $1.20 \times 10^{4}$ & $1.55 \times 10^{4}$ & 1.27 \\
\hline A200 & $>95$ & $2.32 \times 10^{4}$ & $3.53 \times 10^{4}$ & 1.48 \\
\hline R200 & $>95$ & $2.36 \times 10^{4}$ & $3.26 \times 10^{4}$ & 1.27 \\
\hline A500 & $>95$ & $5.78 \times 10^{4}$ & $1.37 \times 10^{5}$ & 1.32 \\
\hline R500 & $>95$ & $5.87 \times 10^{4}$ & $6.28 \times 10^{4}$ & 1.64 \\
\hline
\end{tabular}

\section{General Procedure for the Synthesis of Conventional Free Radical Polymer (FRP) Networks}

To a vial, HEA, AIBN, DSDA and DMF were added. The polymerization was carried out at 65 ${ }^{\circ} \mathrm{C}$ for 24 hours in a vial. While stirring, the solution was sparged with nitrogen gas for $30 \mathrm{~min}$. The vial was placed in an oil bath $65^{\circ} \mathrm{C}$ for $24 \mathrm{~h}$ to perform a conventional FRP reaction. It should 
be noted that it is not possible to control the chain length as it is with RDRP. Therefore, the networks formulations were prepared based on the molar equivalents of monomer to crosslinker. The pre-gel solutions were prepared with the same concentrations as the ATRP and RAFT networks, but without the chain end (neither MBP nor PADTC).

Table S4. The materials used for the synthesis of the four conventional FRP networks with the concentration (molarity [M]) specified. ${ }^{1}$

\begin{tabular}{l|ccc}
\hline Network Molar equiv. & [HEA] & [DSDA] & [AIBN] \\
\hline F100/5 & 4.35 & $2.2 \times 10^{-1}$ & $8.7 \times 10^{-3}$ \\
\hline F200/5 & 4.35 & $1.1 \times 10^{-1}$ & $4.4 \times 10^{-3}$ \\
\hline F500/5 & 4.35 & $4.4 \times 10^{-2}$ & $1.7 \times 10^{-3}$ \\
\hline F500/3 & 4.35 & $2.6 \times 10^{-2}$ & $1.7 \times 10^{-3}$ \\
\hline
\end{tabular}

1) Solvent $=$ DMF; $V_{S}=V_{M}$. 2) The molar equivalents are HEA/DSDA.

Characterization of the Conventional FRP Networks

Table S5. The FRP network formation and structure was compared using the conversion of vinyl bonds, gel fraction, and the SEC results after degradation of parts of the FRP networks, although there were large sections of the gels that did not degrade.

\begin{tabular}{|c|c|c|c|c|}
\hline Network & $\begin{array}{l}\text { Conv. Vinyl } \\
\text { Bonds (\%), }\end{array}$ & Gel Fraction $^{3}$ & $\begin{array}{l}\text { Soluble fraction } \\
\qquad \text { Avg. } M_{n, a p p}^{4}\end{array}$ & $\begin{array}{r}\text { Soluble } \\
\text { fraction } \oplus^{5}\end{array}$ \\
\hline F100/5 & $>95$ & $0.980 \pm 0.003$ & $3.48 \times 10^{4}$ & $2.3 \pm 0.2$ \\
\hline F200/5 & $>95$ & $0.997 \pm 0.006$ & $3.33 \times 10^{4}$ & $1.98 \pm 0.36$ \\
\hline
\end{tabular}




\begin{tabular}{l|lrrr} 
F500/5 & $>95$ & $0.95 \pm 0.01$ & $4.99 \times 10^{4}$ & $2.2 \pm 0.3$ \\
F500/3 & $>95$ & $0.953 \pm 0.002$ & $9.23 \times 10^{4}$ & $2.12 \pm 0.05$ \\
\hline
\end{tabular}

The values reported are averages $(n=3)$ and uncertainty (standard deviation). 1) The conversion of vinyl bonds was determined by ${ }^{1} \mathrm{H}$ NMR. 2) In cases where the conversion is greater than $95 \%$, $>95$ is reported due to the accuracy of the NMR. 3) The fraction of sol + gel $=1.0$. Therefore, the sol fraction $=1.0-$ gel fraction.4) The $M_{n, \text { app }}$ is the $M_{n}$ observed by SEC (DMF). 5) The Đobserved by SEC (DMF).

It should also be noted that, unlike the RAFT and ATRP network samples $(20 \mathrm{mg})$, none of the FRP network samples $(20 \mathrm{mg})$ did fully degraded. There were substantial swollen gel pieces remaining in the vial. The SEC analysis was run on the solution still present in the vials. Therefore, although the observed $\mathrm{D}$ was lower for the F500/5 and F500/3 networks than the A500/5, A500/3, $\mathrm{R} 500 / 5$, and R500/3 networks, it is not necessarily representative of overall network structure.

\section{Rheological Characterization}

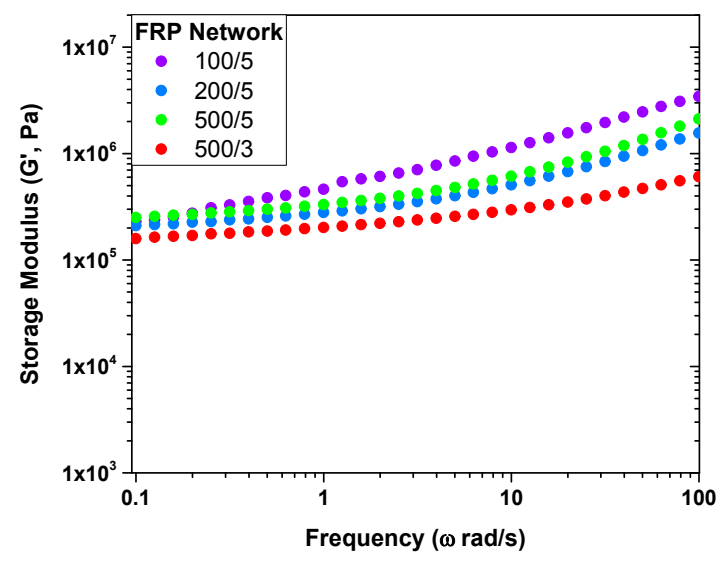

Figure S2. The frequency sweeps for the FRP network samples that show the storage $\left(\mathrm{G}^{\prime}\right)$ : The legend indicates the molar ratios of the monomer/crosslinker. Performed at room temperature 
$\left(25^{\circ} \mathrm{C}\right) ; \gamma=0.1 \%$; applied normal force $2 \mathrm{~N}$. The networks were analyzed after purification and in the dry state.

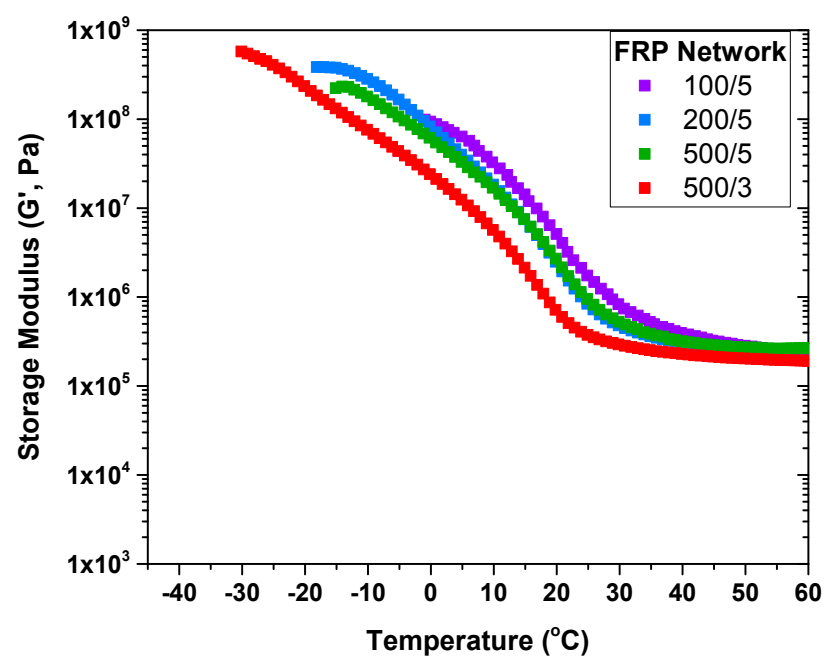

Figure S3. The temperature for the FRP network samples that show the storage modulus $\left(\mathrm{G}^{\prime}\right)$. The legend indicates the molar ratios of the monomer/crosslinker. Temperature Sweeps: heating rate $=$ $2{ }^{\circ} \mathrm{C} / \mathrm{min} ; \gamma=0.1 \% ; \omega=10 \mathrm{rad} / \mathrm{s}$; applied normal force $2 \mathrm{~N}$. The networks were analyzed after purification and in the dry state.

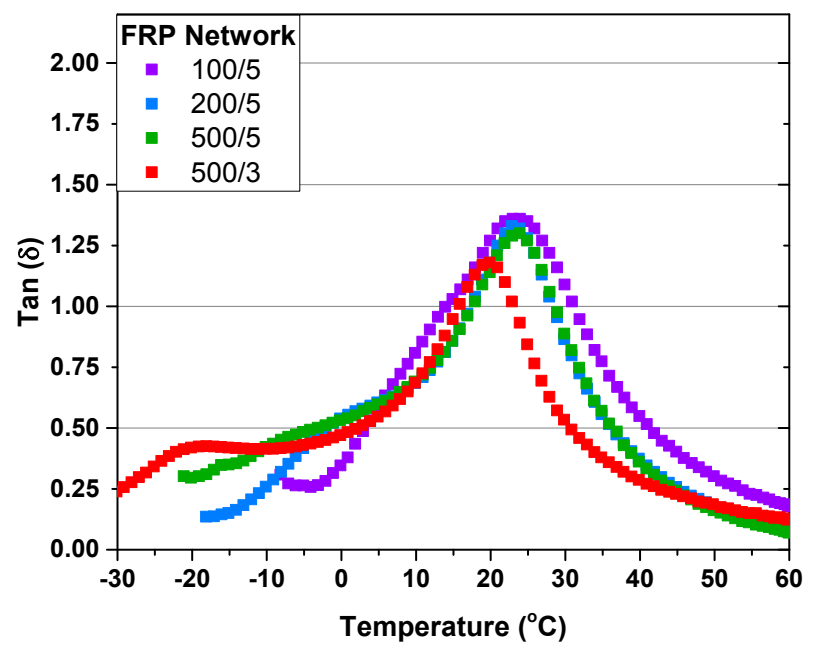


Figure S4. The temperature for the FRP network samples that show the $\tan (\delta)$ profile vs temperature. The legend indicates the molar ratios of the monomer/crosslinker. Temperature sweeps: heating rate $=2^{\circ} \mathrm{C} / \mathrm{min} ; \gamma=0.1 \% ; \omega=10 \mathrm{rad} / \mathrm{s}$; applied normal force $2 \mathrm{~N}$. The networks were analyzed after purification and in the dry state.

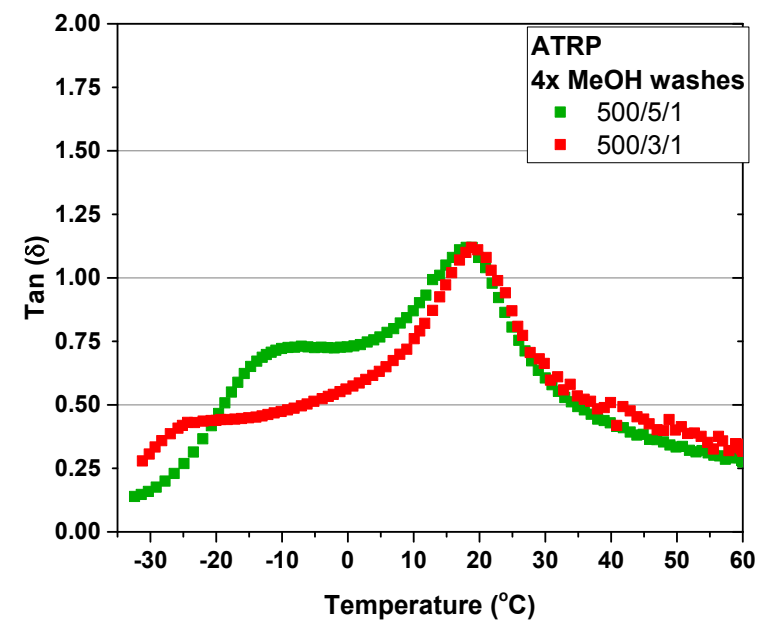

Figure S5. The ATRP network the tan $(\delta)$ vs temperature profile after after only $4 \times \mathrm{MeOH}$ washes. The legend indicates the molar ratios of the monomer/crosslinker/chain end. Temperature Sweeps: heating rate $=2{ }^{\circ} \mathrm{C} / \mathrm{min} ; \gamma=0.1 \% ; \omega=10 \mathrm{rad} / \mathrm{s}$; applied normal force $2 \mathrm{~N}$. The networks were analyzed after purification and in the dry state. 